\title{
Characteristics and Mortality Rates of Patients Following Burn Injury: A Retrospective Series of 1942 Cases
}

\author{
Ayten Saracoglu, MD ${ }^{1}$, Tamer Kuzucuoglu, $\mathrm{MD}^{2}$, Sezer Yakupoglu, $\mathrm{MD}^{2}$, Erhan Tuncay, $\mathrm{MD}^{3}$ \\ Recep Demirhan, Assoc. Prof. MD ${ }^{4}$
}

${ }^{1}$ Department of Anesthesiology and Reanimation, Marmara University Medical School, Istanbul, Turkey

${ }^{2}$ Clinic of Anesthesiology and Reanimation, Kartal Dr. Lutfi Kirdar Training and Research Hospital, Istanbul, Turkey

${ }^{3}$ Clinic of General Surgery, Kartal Dr. Lutfi Kirdar Training and Research Hospital, Istanbul, Turkey

${ }^{4}$ Clinic of Thoracic Surgery, Kartal Dr. Lutfi Kirdar Training and Research Hospital, Istanbul, Turkey

tkuzucuoglu@yahoo.com.tr, sezeryak@gmail.com

erhantuncay@gmail.com, recepdemirhan@hotmail.com

Corressponding Author: Dr. Ayten Saracoglu, Department of Anesthesiology and Reanimation, Marmara University Medical School, Fevzi Cakmak Mh. Ust Kaynarca, Pendik Istanbul, Turkey

Abstract

Objectives: The aim of this study is to analyze the demographic and epidemiologic features of patients admitted to our burn center, to reveal the preventive measures for burn injuries.

Context: Patients were analyzed for age, sex, etiology, type or severity of burn injury, size of burned area, mortality rates, duration of stay at hospital and other associated injuries. Between 2 months and 79 years of age, 1942 burn patients were included.

Results: The types of burn injury were classified as electrical, flame, scalding, contact, chemical burns and others. Scalding burns were most commonly encountered type of burns $(44.74 \%)$. The average percentage of burn surface area was calculated as $15.3 \%$. The rate of mortality was higher for electrical burn injuries (17.1\% of deceased patients) and flame burns ( $69.4 \%$ of deceased patients).

Conclusions: Burn injuries are preventable disasters with undoable physical and psychological scars, therefore prevention and education programs should be performed.

Key words: epidemiology; burn; mortality; injury; intensive care

\section{INTRODUCTION}

Burn injuries continue to be an important health problem in developing countries with mortal consequences which could arise at every age. These injuries with severe physical and psychological outcomes necessitate a prolonged and expensive treatment protocol resulting in a great deal of labour loss [1]. The predisposing factors, the demographical data and the epidemiologic coverage of the burn injuries differ between various countries and different regions. The etiological factors for burn injuries also vary among different countries [2]. The best approach to treat a burn related injury is its prevention [3].

The analysis of the burn injury types admitted to the burn centers in our region constitutes the major contributing factor for the development of effective burn prevention programs. In Turkey patients with acute burn injuries are usually brought to the nearest local hospital first, where the primary interventions take place and the patients are stabilized followed by their transfer to the burn centers. Our facility with a 19 bedded ward and a 6 bedded intensive care unit is the greatest burn center of the Marmara region; it provides burn care services to more than half of all the patients originating from the Marmara region. The other 2 burn centers of this region are Gata Haydarpaşa Military Hospital (12 beds) and Istanbul University Hospital (16 beds).

There are various studies in the literature which were designed to evaluate the epidemiologic features of burn injuries and to determine the specific risk factors in Turkey [4-6]. However these studies were mostly confined to a predefined age group, specific burn 
types or a distinct geographical location. A population based epidemiologic study for Marmara region is not conducted yet and the statistical data for burn cases are still unknown. The aims of this study are to analyze the demographic and epidemiologic features of patients admitted to our burn center, to identify the main etiologic factors and to reveal the preventive measures for burn injuries.

\section{Methods}

Following the approval by our hospitals' local ethics committee the patients admitted to our burn center were analyzed retrospectively. All data were collected from our medical recording system and 1942 burn patients between 2 months and 79 years of age were included in the study. All of the patients were analyzed for the following parameters: age, sex, etiology or type of burn injury, severity of burn injury, size of burned area, mortality rates, duration of stay at the hospital and other associated injuries. In order to determine and compare the risk factors and the prognosis, the patients monitorized in the intensive care unit and the patients followed up in the ward were assigned into two different groups. Outpatient burns were excluded from the study. Accoring to the American Burn Association guidelines the lesions of the patients treated in our burn center on an inpatient basis consisted of moderate and severe burn injuries [7]. The treatment and care of the patients were planned and carried out according to the "Advanced Burn Life Support Protocols of the American Burn Association" [8].

During the treatmentphase 22 patients weretransfered to another hospital thus they were excluded from the study. The Lund method was utilized to calculate the burn surface area [9]. The routine care of burn patients in our burn center includes the application of an appropriate analgesic method, vaccination for tetanus, fluid resuscitation, transfusion of blood and appropriate blood products if needed, daily washing in the burn tanks and appropriate surgical interventions including debridement, fasciotomy and grafting as well as flap coverage.

\section{Statístícal Analysis}

SPSS Windows version 20.0 software was used for the statistical analysis. The descriptive statistics of the data were performed with proportion and frequence values. Chi-square test and Mann-Whitney U test were utilized for the analysis of the variables. Data was expressed as mean \pm standard deviation. $\mathrm{P}<0.05$ was considered statistically significant.

\section{RESULTS}

The average age of the patients was 28.3 (ranged between 2 months and 79 years). The average age of patients monitorized in the intensive care unit was significantly higher than that of the patients in the wards $(30.2 \pm 20.5$ versus $21.4 \pm 21.3$ respectively; $\mathrm{p}<$ 0.05). Among 288 patients below 1 year of age 24 patients were followed up in the intensive care unit (8.3\%). Among 359 patients between 1 and 4 years of age 44 patients were followed up in the intensive care unit (12.3\%). Among 120 patients between 4 and 10 years of age 16 patients were followed up in the intensive care unit (13.3\%). Among 182 patients between 10 and 20 years of age 53 patients were followed up in the intensive care unit (29.1\%). Among 864 patients between 20 and 60 years of age 252 patients were followed up in the intensive care unit (29.2\%). Burn patients between 20 and 60 years of age constitute the greatest proportion among all age groups. And finally 42 out of 142 patients between 60 and 79 years of age were followed up in the intensive care unit (29.6\%). The proportion of patients monitorized in the intensive care unit was significantly greater above 10 years of age. Compared to the ward the male to female ratio was found to be significantly higher in the intensive care unit $(2.13$ versus 3.12 respectively; $\mathrm{p}=0.002$ ).

The duration of stay at the hospital was significantly higher among the ward patients (12.8 \pm 11.2 days $)$ in comparison to the patients monitorized in the intensive care unit $(8.9 \pm 12.8$ days $)(p<0.05)$. The average percentage of burn surface area was calculated as $15.3 \%$. The burn percentage was significantly higher in the intensive care unit $(38.1 \pm 22.7 \%)$ compared to the ward patients $(11.9 \pm 10.7 \%)$ ( $p<0.05)$. Approximately $58 \%$ of the patients monitorized in the intensive care unit $(n=189)$ had a burn percentage between $25 \%$ and $50 \%$. The majority of the patients followed up in the wards $(92.9 \%, \mathrm{n}=1804)$ had a burn percetage of $0-15 \%$.

The types of burn injury were classified as electrical burns, flame burns, scalding burns, contact burn injuries, chemical burns and others. Scalding burns were found to be the most commonly encountered type of burn injury (44.74\%). Scalding burn injuries 
Characteristics and Mortality Rates of Patients Following Burn Injury: A Retrospective Series of 1942 Cases

mostly resulted from accidents occured at home $(74.2 \%)$ and $90.6 \%$ of these patients were followed up at the ward. Flame burns were the most frequently encountered etiology for the patients requiring close monitorization at the

intensive care unit (52.21\%). For patients with the electrical burn and flame burn injuries the rate of admission to the intensive care unit was significantly higher than other types of burn injuries $(\mathrm{p}<0.05$, Table 1).

Table 1. Patient demographics, the duration of hospital stay, burn percentage and causes of burn injuries

\begin{tabular}{|c|c|c|c|c|}
\hline & & Intensive Care Unit & Ward & $\mathbf{p}$ \\
\hline Age (years) & & $30.2 \pm 20.5$ & $21.4 \pm 21.3$ & $<0.001$ \\
\hline \multirow{6}{*}{ Age groups (years) } & $0-1$ & $\mathrm{n}=24(8.3 \%)$ & $\mathrm{n}=264(91.7 \%)$ & \\
\hline & $1-4$ & $\mathrm{n}=44(12.3 \%)$ & $\mathrm{n}=315(87.7 \%)$ & \\
\hline & $4-10$ & $\mathrm{n}=16(13.3 \%)$ & $\mathrm{n}=104(86.7 \%)$ & \\
\hline & $10-20$ & $\mathrm{n}=53(29.1 \%)$ & $\mathrm{n}=129(70.9 \%)$ & \\
\hline & $20-60$ & $\mathrm{n}=252(29.2 \%)$ & $\mathrm{n}=612(70.8 \%)$ & \\
\hline & $60-100$ & $n=42(29.6 \%)$ & $\mathrm{n}=100(70.4 \%)$ & \\
\hline \multirow{2}{*}{ Gender } & Female & $\mathrm{n}=105(24.2 \%)$ & $\mathrm{n}=482(31.9 \%)$ & \multirow{2}{*}{0.002} \\
\hline & Male & $\mathrm{n}=328(75.8 \%)$ & $n=1027(68.1 \%)$ & \\
\hline Hospital stay (days) & & $8.9 \pm 12.8$ & $12.8 \pm 11.2$ & $<0.001$ \\
\hline Burn percentage (\%) & & $38.1 \pm 22.7$ & $11.9 \pm 10.7$ & $<0.001$ \\
\hline \multirow{5}{*}{ Burn percentage $(\%)$} & $0-15$ & $\mathrm{n}=85(7.1 \%)$ & $\mathrm{n}=1114(92.9 \%)$ & \\
\hline & $15-25$ & $\mathrm{n}=65(19.2 \%)$ & $\mathrm{n}=273(80.8 \%)$ & \\
\hline & $25-50$ & $\mathrm{n}=189(58.0 \%)$ & $\mathrm{n}=137(42.0 \%)$ & \\
\hline & $50-75$ & $\mathrm{n}=55(84.6 \%)$ & $\mathrm{n}=10(15.4 \%)$ & \\
\hline & $75-100$ & $\mathrm{n}=39(97.5 \%)$ & $\mathrm{n}=1(2.5 \%)$ & \\
\hline \multirow{5}{*}{ Cause of burn injury } & Electrical & $n=102(44.2 \%)$ & $\mathrm{n}=129(55.8 \%)$ & $<0.001$ \\
\hline & Flame & $\mathrm{n}=224(35.8 \%)$ & $\mathrm{n}=402(64.2 \%)$ & $<0.001$ \\
\hline & Chemical & $\mathrm{n}=5(11.9 \%)$ & $\mathrm{n}=37(88.1 \%)$ & \\
\hline & Contact & $\mathrm{n}=7(9.9 \%)$ & $\mathrm{n}=64(90.1 \%)$ & \\
\hline & Scalding & $\mathrm{n}=82(9.4 \%)$ & $\mathrm{n}=787(90.6 \%)$ & \\
\hline
\end{tabular}

During the study a total of 35 female and 100 male patients passed away (mortality rate: $7.47 \%$ ). The average age of the patients who died was significantly higher than the rest of the patients $(37.1 \pm 21.9$ years of age versus $22.4 \pm 21.1$ years of age respectively, $\mathrm{p}<$ 0.05). The distribution of gender characterictics were similar in both groups $(\mathrm{p}=0.259)$. The duration of stay at the burn center was significantly shorter in the group of patients who passed away $(9.8 \pm 16.6$ days versus $12.1 \pm 11.2$ days; $p<0.05$ ). The burn percentage was significantly higher among the deceased patients (55.4 $\pm 22.7 \%$ versus $14.9 \pm 13.9 \%$; $p<0.05$ ). The burn percentage was found to have a positive corelation with the mortality risk. The rate of mortality was significantly higher for electrical burn injuries $(17.1 \%$ of all deceased patients) and flame burns $(69.4 \%$ of all deceased patients) compared to the other types of burn injuries $(p<0.05$, Table 2$)$. 
Characteristics and Mortality Rates of Patients Following Burn Injury: A Retrospective Series of 1942 Cases

Table 2. the relation between mortality rates and patient demographics, the duration of hospital stay, burn percentage and causes of burn injuries

\begin{tabular}{|c|c|c|c|c|}
\hline & & Alive & Dead & $\mathbf{p}$ \\
\hline Age (years) & & $22.4 \pm 21.1$ & $37.1 \pm 21.9$ & $<0.001$ \\
\hline \multirow{6}{*}{ Age groups (years) } & $0-1$ & $\mathrm{n}=17(70.8 \%)$ & $\mathrm{n}=7(29.2 \%)$ & \\
\hline & $1-4$ & $\mathrm{n}=41(93.2 \%)$ & $\mathrm{n}=3(6.8 \%)$ & \\
\hline & $4-10$ & $\mathrm{n}=13(81.3 \%)$ & $\mathrm{n}=3(18.8 \%)$ & \\
\hline & $10-20$ & $\mathrm{n}=34(64.2 \%)$ & $\mathrm{n}=19(35.8 \%)$ & \\
\hline & $20-60$ & $\mathrm{n}=173(68.7 \%)$ & $\mathrm{n}=79(31.3 \%)$ & \\
\hline & $60-100$ & $\mathrm{n}=18(42.9 \%)$ & $\mathrm{n}=24(57.1 \%)$ & \\
\hline \multirow{2}{*}{ Gender } & Female & $\mathrm{n}=552(23.5 \%)$ & $\mathrm{n}=35(25.9 \%)$ & \multirow{2}{*}{0.259} \\
\hline & Male & $n=1255(76.5 \%)$ & $\mathrm{n}=100(74.1 \%)$ & \\
\hline Hospital stay (days) & & $12.1 \pm 11.2$ & $9.8 \pm 16.6$ & 0.001 \\
\hline Burn percentage (\%) & & $14.9 \pm 13.9$ & $55.4 \pm 22.7$ & $<0.001$ \\
\hline \multirow{5}{*}{ Burn percentage (\%) } & $0-15$ & $\mathrm{n}=80(94.1 \%)$ & $n=5(5.9 \%)$ & \\
\hline & $15-25$ & $\mathrm{n}=57(87.7 \%)$ & $\mathrm{n}=8(12.3 \%)$ & \\
\hline & $25-50$ & $\mathrm{n}=134(70.9 \%)$ & $\mathrm{n}=55(29.1 \%)$ & \\
\hline & $50-75$ & $\mathrm{n}=21(38.2 \%)$ & $\mathrm{n}=34(61.8 \%)$ & \\
\hline & $75-100$ & $\mathrm{n}=6(15.4 \%)$ & $\mathrm{n}=33(84.6 \%)$ & \\
\hline \multirow{6}{*}{ Cause of burn injury } & Electrical & $\mathrm{n}=533(85.1 \%)$ & $n=93(14.9 \%)$ & $<0.001$ \\
\hline & Flame & $\mathrm{n}=208(90.0 \%)$ & $\mathrm{n}=23(\mathbf{1 0 . 0 \% )}$ & 0.047 \\
\hline & Chemical & $\mathrm{n}=40(95.2 \%)$ & $\mathrm{n}=2(4.8 \%)$ & \\
\hline & Contact & $\mathrm{n}=856(98.5 \%)$ & $n=13(1.5 \%)$ & \\
\hline & Scalding & $\mathrm{n}=71(100.0 \%)$ & $\mathrm{n}=0(0.0 \%)$ & \\
\hline & Other & $\mathrm{n}=121(97.6 \%)$ & $\mathrm{n}=3(2.4 \%)$ & \\
\hline
\end{tabular}

The duration of stay at the hospital was burns and flame burn injuries had a significantly significantly higher in the electrical burn injuries (14.4 \pm 17.3 days) and flame burns (14.3 \pm 12.7 days) compared to the other types of burn injuries $(\mathrm{p}<0.05)$. Similarly, the patients with electrical higher burn percentage in comparison to the patients with other types of burn injuries $(21.1 \pm$ $17.7 \%$ and $26.5 \pm 22.3 \%$ respectively; $\mathrm{p}<0.05$ ) (Table 3).

Table 3. The relation between the causes of burn injuries and the duration of hospital stay with burn percentage

\begin{tabular}{|l|l|l|l|l|}
\hline Cause of burn injury & Hospital stay (days) & $\mathbf{p}$ & Burn percentage (\%) & $\mathbf{p}$ \\
\hline Flame & $14.3 \pm 12.7$ & $<\mathbf{0 . 0 0 1}$ & $26.5 \pm 22.3$ & $<\mathbf{0 . 0 0 1}$ \\
\hline Electrical & $14.4 \pm 17.3$ & $<\mathbf{0 . 0 0 1}$ & $21.1 \pm 17.7$ & $<\mathbf{0 . 0 0 1}$ \\
\hline Scalding & $10.8 \pm 9.3$ & & $12.6 \pm 11.8$ & \\
\hline Contact & $8.9 \pm 8.6$ & & $9.7 \pm 8.6$ & \\
\hline Chemical & $9.0 \pm 8.5$ & & $13.6 \pm 14.8$ & \\
\hline Other & $7.2 \pm 6.9$ & & $8.3 \pm 12.1$ & \\
\hline
\end{tabular}




\section{Díscussion}

The aim of this study was to determine the distribution and the importance of traumatic burn injuries occuring in Marmara region. One of the most important problems of the burn victims is the increased risk for additional complications to develop as a result of prolonged hospital stay. The loss of extremity, opportunistic infections, frequent surgical interventions, aesthetic deformations and economic deprivations are other major accompanying complications of the burn injuries. Thus it is quite important to take action before burn injury develops. Enough data should be collected in our society about burn related injuries and the awareness of the general population should be improved with educational programs.

In the developed as well as the developing countries the burn injury itself is a major reason for mortality [10]. Turkey still has the status of a developing country which requires a special attention on burn injuries. Fortunately in recent years progress was made on the burn epidemiology and the management of statistical data in Turkey. Examples for this progress include the studies evaluating the burn injuries related to the traditional turkish cooking methods [11] and the reports of mortality rates from various burn centers [12].

In our study adult male patients constituted the majority of patients admitted to our burn center. This finding was similar to various other study results $[13,14]$. However, female patients $[15,16]$ and children below 10 years of age [17] were reported as the predominant burn patient groups in some other studies. According to our data scalding burns represented the greatest percentage of burn cases in our study. The results of many other studies also supported this finding $[18,19]$. On the other hand Xie et al. [20] revealed in their series that flame burns constituted the majority of burn injuries. In our study scalding burns were followed by flame burns and electrical burn injuries as the second and third most frequent types of burn injury. Our burn center is located close to the industrial zone which explains the relatively increase frequences of flame burns, electrical burns and contact burn injuries.

According to our findings the most common location of scalding burn injuries was home. An important factor facilitating this type of burn injuries is the the crowded family structure with multiple small children as observed in the families which had been originally migrated from the easternmost part of Turkey. The neglectful attitudes of parents towards their children at home are another important etiological factor for burn accidents with quite unfavorable end results. Thus scalding type of burn injuries via hot water, tea, milk or soup come occur more frequently than usual. The traditional turkish way of life imposes the consumption of slops followed by hot tea thus the risk for burn injury obviously increases in the evening when all of the family members are present at home. In addition, at rural areas milk and milk derivatives are not bought as pasteurized products but the parents usually milk their cow and then boil the milk before serving to their children, which constitutes another risk factor for the occurence of scalding burns [21] On the other hand some parents make their older children responsible of the smaller sibling at home, obviously a clear invitation to many major accidents including burn injuries.

The highest mortality rate among our cases was observed in the flame burns group, followed by the electrical burn injuries. The level of burn percentage showed a direct correlation with the mortality rate. The highest burn percentage was noted in the flame burns group and the longest duration of hospitalization in the electrical burn injuries group. Our mortality ratio was calculated to be around $7 \%$ which was much lower than various other study results. A possible explanation for this finding was speculated to be the average burn percentage of $15.3 \%$ in this study. On the other hand the mean burn percentage of deceased patients were calculated as $55.4 \%$ whereas the mean burn percentage of rest of the patients was found to be $14.9 \%$. Among the flame burn patients who have the highest burn percentage levels the duration of hospital stay was found to be increased in addition to an obvious increase in the mortality rates.

In Turkey a society called Burn and Fire Disasters Association exists, which is based in Ankara and founded by medical doctors. This association was established to conduct studies, education programs, national and international scientific activities on burns and fire disasters; it is still actively pursuing its preventive and educational targets. We believe that the conduction of appropriate notifications and education programs will provide major benefits to protection from accidents. Children are actually 
Characteristics and Mortality Rates of Patients Following Burn Injury: A Retrospective Series of 1942 Cases

without prejudice and quite easy to educate thus they should continue to be regarded as a vital part of the preventive programs [22].

\section{ConClusion}

This study revealed that the majority of burn patients in our region consisted of middle aged male patients. The type of burn injury with the highest mortality risk was found to be the flame burns. The most common type of injury was noted as scalding burns and the largest group of patients experienced the burn injury while actively cooking in the evening. We believe that the traditional methods for cooking and tea brewing in Turkey should be re-evaluated and necessary precautions have to be undertaken. Burn injuries are preventable disasters with undoable physical and psychological scars, thus the efforts for preventive measures and education programs should be increased. All social assistance foundations and burn associations should also work toward this goal.

\section{REFERENCES}

[1] Ciftçi I, Arslan K, Altunbaş Z, Kara F, Yilmaz H. Epidemiologic evaluation of patients with major burns and recommendations for burn prevention. Ulus Travma Acil Cerrahi Derg 2012, 18:105-10.

[2] Taghavi M, Rasouli MR, Boddouhi N, Zarei MR, Khaji A, Abdollahi M. Epidemiology of outpatient burns in Tehran: an analysis of 4813 cases. Burns 2010, 36:109-13.

[3] Sadeghi-Bazargani H, Mohammadi R. Epidemiology of burns in Iran during the last decade (20002010): review of literature and methodological considerations. Burns 2012, 38:319-29.

[4] Albayrak Y, Cakır C, Albayrak A, Aylu B. A comparison of the morbidity and mortality of tandir burns and non-tandir burns: experience in two centers. Ulus Travma Acil Cerrahi Derg 2011, 17:323-8.

[5] Yavuz A, Ayse A, Abdullah Y, Belkiz A. Clinical and demographic features of pediatric burns in the eastern provinces of Turkey. Scand J Trauma Resusc Emerg Med 2011, 19:6.

[6] Serinken M, Türkçüer I, Karcıoğlu Ö, Akkaya S, Uyanık E. Domestic accidents involving women and first aid knowledge. Ulus Travma Acil Cerrahi Derg 2011, 17:445-9.
[7] Gibran NS; Committee on Organization and Delivery of Burn Care, American Burn Association. Practice Guidelines for burn care, 2006. J Burn Care Res 2006, 27:437-8.

[8] Iqbal T, Saaiq M, Ali Z. Epidemiology and outcome of burns: Early experience at the country's first national burns Centre. Burns 2013, 39:358-62.

[9] Panjeshahin MR, Lari AR, Talei A, Shamsnia J, Alaghehbandan R. Epidemiology and mortality of burns in the South West of Iran. Burns 2001, 27:219-26.

[10] Hashmi M, Kamal R. Management of patients in a dedicated burns intensive care unit (BICU) in a developing country. Burns 2013, 39:493-500.

[11] Al B, Coban S, Güloğlu C. Tandir burns in and around Diyarbakir, Turkey. Ulus Travma Acil Cerrahi Derg 2010, 16:59-62.

[12] Al B, Yildirim C, Coban S, Aldemir M, Güloğlu C. Mortality factors in flame and scalds burns: our experience in 816 patients. Ulus Travma Acil Cerrahi Derg 2009; 15:599-606.

[13] Chien WC, Pai L, Lin CC, Chen HC. Epidemiology of hospitalized burns patients in Taiwan. Burns 2003, 29:582-8.

[14] Jie X, Baoren C. Mortality rates among 5321 patients with burns admitted to a burn unit in China: 1980-1998. Burns 2003, 29:239-45.

[15] Othman N, Kendrick D. Burns in Sulaymaniyah province, Iraq: epidemiology and risk factors for death in patients admitted to hospital. J Burn Care Res 2011, 32:e126-34.

[16] Morita S, Higami S, Yamagiwa T, Iizuka S, Nakagawa Y, Yamamoto I, et al. Characteristics of elderly Japanese patients with severe burns. Burns 2010, 36:1116-21.

[17] Agbenorku P, Edusei A, Ankomah J. Epidemiological study of burns in Komfo Anokye Teaching Hospital, 2006-2009. Burns 2011, 37:1259-64.

[18] Alaghehbandan R, MacKay Rossignol A, Rastegar Lari A. Pediatric burn injuries in Tehran, Iran. Burns 2001, 27:115-8.

[19] Zhou B, Zhou X, Ouyang LZ, Huang XY, Zhang PH, Zhang $\mathrm{MH}$, et al. An epidemiological analysis of paediatric burns in urban and rural areas in south central China. Burns 2014, 40:150-6. 
Characteristics and Mortality Rates of Patients Following Burn Injury: A Retrospective Series of 1942 Cases

[20] Xie B, Xiao SC, Peng XD, Zhu SH, Lv KY, Li HY, et al. Epidemiology and outcome analysis of severe extensive burns: a 12-year summary of 103 cases in a burn center in China. J Burn Care Res 2012, 33:e127-32.

[21] Koç Z, Sağlam Z. Burn epidemiology and cost of medication in paediatric burn patients. Burns 2012, 38:813-9.

[22] Lari AR, Alaghehbandan R, Nikui R. Epidemiological study of 3341 burns patients during three years in Tehran, Iran. Burns 2000, 26:49-53.

Citation: Ayten Saracoglu, Tamer Kuzucuoglu, Sezer Yakupoglu, Erhan Tuncay, Recep Demirhan, Assoc. Prof. Characteristics and Mortality Rates of Patients Following Burn Injury: A Retrospective Series of 1942 Cases. Open Access Journal of Internal Medicine. 2018; 1(1): 14-20.

Copyright: (C) 2018 Ayten Saracoglu, Tamer Kuzucuoglu, Sezer Yakupoglu, Erhan Tuncay, Recep Demirhan, Assoc. Prof. This is an open access article distributed under the Creative Commons Attribution License, which permits unrestricted use, distribution, and reproduction in any medium, provided the original work is properly cited. 